\title{
Neue Mehrheiten in Bayern
}

\author{
Es kommt zum Machtwechsel in der Kassenzahnärztlichen Vereinigung Bayerns (KZVB). Der \\ FVDZ Bayern und die regionalen Vereine sind die Wahlsieger 2016. Die drei Listen mit Kandida- \\ ten des FVDZ Bayern und regionaler Vereine werden eine Fraktionsgemeinschaft bilden und \\ stellen damit 16 der künftig 27 Delegierten in der Vertreterversammlung (58,9 Prozent). Die Ära \\ des bisherigen KZVB-Chefs Dr. Janusz Rat geht damit nach zwölf Jahren zu Ende.
}

Die Fraktionsgemeinschaft der Kandidaten des FVDZ und regionaler Vereine setzt sich mit insgesamt 16 Sitzen aus den folgenden Listen zusammen:

- Liste 2: Kandidaten des FVDZ und regionaler Vereine aus Mittelfranken, Oberfranken und Unterfranken mit dem Spitzenkandidaten Dr. Rüdiger Schott: 8 Sitze

- Liste 3: Kandidaten des FVDZ und regionaler Vereine in München (Stadt und Land) und Schwaben mit dem Spitzenkandidaten Christian Berger: 4 Sitze

- Liste 4: Kandidaten des FVDZ und regionaler Vereine aus Oberbayern, Oberpfalz und Niederbayern mit dem Spitzenkandidaten Dr. Michael Rottner: 4 Sitze.

Zukunft Zahnärzte Bayern (ZZB) konnte nur 10 Sitze erringen (34,5 Prozent). 2010 waren es bei 24 Delegierten noch 13 Sitze. Der bisherige Versammlungsleiter der Vertreterversammlung, Dr. Günter Schneider, hat es nicht mehr auf einen Listenplatz geschafft und wird in der Vertreterversammlung ab 2017 fehlen. Ein Sitz geht an das Team Bayern von Dr. Klaus Kocher.

Mit diesem Wahlergebnis, bei einer Wahlbeteiligung von etwa 45 Prozent, ist die zwölfjährige Dominanz von ZZB in der KZVB beendet. Bereits Mitte 2015 hatte die krisengeschüttelte Fraktion von Dr. Janusz Rat ihre hauchdünne Mehrheit in der Vertreterversammlung verloren. Anfang 2016 löste KZVB-Vize Dr. Stefan Böhm Dr. Rat als Landesvorsitzender von ZZB ab, nur um wenige Monate später von seiner eigenen Partei zum Rücktritt gezwungen $\mathrm{zu}$ werden. Bis zu diesem Bruch war Böhm der neue Spitzenkandidat und Hoffnungsträger von ZZB für die KZV-Wahl.

\section{Zusammenarbeit mit allen VV-Delegierten}

Die Fraktionsgemeinschaft von FVDZ und Vereinen wird zwar eine komfortable Mehrheit in der künftigen Vertreterversammlung aufweisen, dennoch setzt der Landesvorsitzende des FVDZ Bayern, Christian Berger, einer Pressemeldung zufolge aufZusammenarbeit mit allen Delegierten der künftigen VV. Die bisher fehlende Einbindung der fast gleichstarken Opposition habe der FVDZ Bayern in der von ZZB-dominierten Vertreterversammlung wiederholt kritisiert. „Wir werden unsere Wahlversprechen einlösen und mit allen zusammenarbeiten, die gewillt sind, sich konstruktiv einzubringen. Das Wählervotum hat gezeigt, dass unsere Forderungen nach mehr Zusammenarbeit der beiden Körperschaften KZVB und BLZK, mehr Transparenz in der KZVB-Vorstandsarbeit und mehr Demokratie und Mitsprache von den bayerischen Zahnärztinnen und Zahnärzten mitgetragen werden", so Berger.

Der scheidende KZVB-Chef Rat hat die Niederlage eingeräumt. Er gratulierte dem FVDZ Bayern zu seinem Wahlerfolg und wünscht ihm eine glückliche Hand für die „großen Herausforderungen, die vor ihm liegen“. Er habe zusammen mit seinen Mitstreitern von ZZB in den vergangenen zwölf Jahren das Bestmögliche für die bayerischen Vertragszahnärzte erreicht und hinterlasse ein gut bestelltes Haus. Wenn die Wähler sich für einen Wechsel entschieden hätten, müsse man das respektieren, so Rat.

\section{Künftige Opposition gratuliert}

Gratulation und das Angebot zur konstruktiven Zusammenarbeit kommen auch von der künftigen Opposition. Der stellvertretende ZZB-Vorsitzende Dr. Cornelius Haffner sagte mit Blick auf die Gerichtstreitigkeiten, die vom KZVB-Vorstand im vergangenen Jahr initiiert worden waren: „Wenn wir auch in $\mathrm{Zu}$ kunft weiter Gerichte bemühen müssen, um im politischen Umgang miteinander Klarheit zu schaffen, dürfen wir uns nicht wundern, wenn wir uns als Wählervertreter immer weiter von der bayerischen Zahnärzteschaft entfernen“.

Anita Wuttke, freie Journalistin

\section{Hier steht eine Anzeige.}

